MIT-CTP-3454

NSF-KITP-03-111

hep-th/0312172

\title{
Brane/Flux Interactions in Orientifolds ${ }^{1}$
}

\author{
Marcus Berg*, Michael Haack*, and Boris Körs ${ }^{\dagger}$ \\ * Kavli Institute for Theoretical Physics \\ University of California \\ Santa Barbara, CA 93106-4030, USA \\ mberg,mhaack@kitp.ucsb.edu \\ ${ }^{\dagger}$ Center for Theoretical Physics \\ Laboratory for Nuclear Science \\ and Department of Physics \\ Massachusetts Institute of Technology \\ Cambridge, MA 02139, USA \\ kors@lns.mit.edu
}

\begin{abstract}
Combining the benefits of D-branes and background fluxes in string compactifications opens up the possibility to explore phenomenologically interesting brane world models with stabilized moduli. However, it is difficult to determine interaction effects among open strings and fluxes in the effective action. We derive the full bosonic Lagrangian of a (spontaneously broken) $\mathcal{N}=4$ supersymmetric model with D3-branes and NSNS and RR 3-form fluxes in an orientifold of type IIB, that, without fluxes, would be T-dual to type I theory. In the limit where backreaction in form of a warp factor is neglected, the effective action can be obtained through a procedure that combines dimensional reduction and T-duality, and it is found to be in agreement with results from gauged supergravity. This provides evidence for the consistency of this commonly used approximation scheme.
\end{abstract}

\section{Introduction}

It has become evident that fluxes, i.e. vacuum expectation values for certain higher rank tensor fields, can provide a step towards solving the moduli problem of string compactification (see 2, 3, 4, for the first appearances of fluxes in string theory). In the effective four-dimensional field theory, they induce a scalar potential and mass terms for many of the otherwise massless scalar fields, that parametrize the size and shape of the compactification space. On the other hand, in order to introduce non-abelian gauge symmetries into models within type II or type I string theories one has to add D-branes as well. In the absence of fluxes, these wrap calibrated supersymmetric cycles of the background geometry, and the interactions of open and closed string modes are largely dictated by supersymmetry and anomaly considerations. Once fluxes are turned on, it is well known that this simple picture may be drastically modified, as happens in the dielectric effect [5]. The effective action with fluxes is given by a suitably gauged version of the original supergravity. On the other hand, since the presence of D-branes induces an effective scalar potential through the brane tension, the known scalar potential for fluxes without

\footnotetext{
${ }^{1}$ The present article is based on the work [1].
} 
branes is modified. We address the issue of flux/brane interaction effects in the context of a simplified framework [6, 7], an $\mathcal{N}=4$ supersymmetric orientifold compactification of type IIB with 3-form NSNS and RR fluxes and space-time filling D3-branes, by deriving the full bosonic Lagrangian of the effective theory. This will involve the standard gauge theory for the D3-branes and the bulk supergravity including the 3-form fluxes, their Chern-Simons (CS) interactions as known for coupled supergravity (SUGRA) and super Yang-Mills (YM) systems, and finally extra interaction terms that stem from the nonabelian generalization of the effective Born-Infeld (BI) brane action, as given in [5]. The resulting Lagrangian can be compared in great detail to results of gauged supergravity [8], which confirms the consistency of our approach.

In order to present the model we are considering [6, 7], we first have to explain two ingredients of importance. $i$ ) The well known no-go theorems (see e.g. [3]) for compactifications of ten-dimensional supergravity theories prohibit, under certain circumstances, solutions to the ten-dimensional equations of motion in type II string theory with background fluxes. Following [9] this can be circumvented in orientifold models, which involve (non-dynamical) orientifold planes (Op-planes) with negative energy densities but no perturbative degrees of freedom. $i i$ ) The orientifold model at hand can be understood as a T-dual version of type I string theory compactified on a six-torus $\mathbb{T}^{6}$, T-dualized along all six circles. One may therefore be surprised that it is possible to turn on NSNS and RR 3-form fluxes, since neither the original nor the T-dual model possess the two types of 2-form potentials as dynamical degrees of freedom. We shall explain in which sense the deformation is possible in the T-dual model, though type I only has the RR 2-form potential $C_{2}$ in the spectrum.

\subsection{Orientifolds with fluxes and branes}

The maybe simplest version of the no-go theorems is obtained from the four-dimensional components of the ten-dimensional Einstein equations. If we adopt a general warped ansatz for the metric, $d s_{10}^{2}=\Delta^{-1}\left(x^{i}\right) d s_{4}^{2}\left(x^{\mu}\right)+\Delta^{b}\left(x^{i}\right) d s_{6}^{2}\left(x^{i}\right)$, and specify $d s_{4}^{2}$ to Minkowski space, we find a relation [3]

$$
g^{\mu \nu} R_{\mu \nu} \propto g^{i j} \nabla_{i}\left(\Delta^{2(b-1)} \partial_{j} \ln (\Delta)\right) \propto \mathcal{T}_{\mu \nu} g^{\mu \nu}-\mathcal{T}_{i j} g^{i j}
$$

Any type of matter or energy that satisfies the strong energy condition, as background fluxes and D-brane tension do, gives a negative definite contribution to the right-handside. Integrating the total derivative on the left-hand-side then requires fluxes to vanish and D-branes to be absent. In other words, there are no compact solutions with warped four-dimensional Minkowski (or de Sitter) vacua in type II string theory. There are various ways to circumvent this result, such as higher curvature corrections to the ten-dimensional action or the option of breaking maximal four-dimensional symmetry. Based on the fact that O-planes formally carry negative tension, the most straightforward way to evade the theorem appears to be orientifold-, i.e. type I, compactifications or their non-perturbative lift to F-theory. In this sense, orientifolds are the unique perturbative string models with a compact internal space, that allow simultaneously for fluxes, non-trivial warp factors and D-branes. It actually turns out that the structure of the constraints still strictly prohibits a positive four-dimensional cosmological constant within the framework of static metrics of the type we consider, so only Minkowski or anti-de Sitter solutions exist [9]. 
There are, however, proposals for getting de Sitter backgrounds within string theory, e.g. by considering non-perturbative modifications of the four-dimensional effective action and anti-D3-branes [10].

An important feature of the ten-dimensional solutions is the warp factor $\Delta\left(x^{i}\right)$ in front of the four-dimensional metric. On the one hand, it is very attractive in providing an extremely rich phenomenology in the spirit of Randall-Sundrum brane worlds, on the other hand the moduli space for warped compactifications is not known in general and a dimensional reduction including its effects therefore so far impossible. The usual recipe is to neglect the warp factor in the reduction to four dimensions. Following the arguments given in [9], we assume that the warp factor scales like $\Delta\left(x^{i}\right)=1+\mathcal{O}\left(1 / R^{2}\right)$ and is negligible in the large volume limit $R / \sqrt{\alpha^{\prime}} \rightarrow \infty$. Requiring the overall average radius $R$ to be large compared to the string length $\sqrt{\alpha^{\prime}}$ has the additional benefit that it leads to a separation of the characteristic mass scales, schematically 6

$$
\left.\left.\left.\frac{1}{\sqrt{\alpha^{\prime}}}\right|_{\text {string }} \gg \frac{1}{R}\right|_{\mathrm{KK}} \gg \frac{\alpha^{\prime}}{R^{3}}\right|_{3-\text { flux }} \text {. }
$$

Summarising, we really reduce on a direct product $\mathbb{R}^{4} \times \mathcal{M}^{6}$ and treat the branes and fluxes as a small perturbation on the geometry.

\subsection{The model: type $\mathrm{I}^{\prime}$ on $\mathbb{T}^{6}$}

The definition of type I string theory as an orientifold of type IIB implies that its effective action, with all open string fields set to zero, is obtained by projecting out all states from the type IIB Lagrangian that are odd under the world sheet parity $\Omega$. This leaves a bosonic spectrum in ten-dimensional type I consisting of $\left\{g_{I J}, \Phi, C_{2}, A_{I}^{a}\right\}$, the metric, dilaton and RR 2-form, plus the open string vector fields. Their coupling to the other fields is actually fixed by supersymmetry and for low energies takes the standard form of the type I plus BI Lagrangian. As a trivial remark, all fields of type IIB that are odd under $\Omega$ have to vanish identically.

We can now imagine to compactify on $\mathbb{T}^{6}$ and apply six T-dualities to the theory, which effectively means, we project out type IIB with the T-dual world sheet parity $\Omega^{\prime}=\Omega \Theta(-1)^{F_{L}}$, where $\Theta$ is a reflection of all six circles [6]. This leaves us with the spectrum $\left\{g_{\mu \nu}, g_{i j},\left(B_{2}\right)_{i \mu},\left(C_{2}\right)_{i \mu}, \tau,\left(C_{4}\right)_{i j k l}, A_{\mu}^{a}, A_{i}^{a}\right\}$, the four-dimensional external and sixdimensional internal metric, twelve KK vectors from the NSNS and RR 2-forms, the complex dilaton $\tau=e^{-\Phi}+i C_{0}$, and the open string fields split into internal scalar components and external vector fields. The important observation now is that despite the absence of dynamical 2-form potentials in the spectrum, the background fields of type IIB do not have to vanish, but only be anti-symmetric under $\Theta$, since $f\left(x^{i}\right)=-f\left(-x^{i}\right)$ does not imply $f\left(x^{i}\right)=0$. We can then keep the background values for the field strengths $d B_{2}, d C_{2}$ of the IIB Lagrangian as deformations of the T-dual theory. These are 3-form fluxes in IIB, but since there are no 2 -form potentials in the T-dual theory, they appear as new parameters in the Lagrangian, their form and systematics inherited from the IIB parent theory. A priori, it does not appear guaranteed that this procedure leads to a consistent, supersymmetric theory at all. One purpose of our investigation is to establish this consistency by matching the action with a gauged supergravity Lagrangian. The result will be an explicit and complete bosonic action for the T-dual theory, which we call type 
$\mathrm{I}^{\prime}$, compactified on $\mathbb{T}^{6}$ with NSNS and RR (i.e. complex) 3-form flux, coupled to D3-brane world volume vectors and coordinate scalar fields, thus with $\mathcal{N}=4$ supersymmetry. The steps to perform are: First, we T-dualize type I along six circles to deduce the T-dual CS couplings to the open string fields from the known form in type I. Second, we investigate the non-abelian Born-Infeld action in the form given by Myers [5] for extra contributions. Third, we compare with the $\Omega^{\prime}$-projected IIB Lagrangian to deduce the extra deformations of the Lagrangian due to 3 -form fluxes. The objects of greatest interest are the scalar potential that arises from the combination of fluxes and branes, and the CS action. It is possible to derive the full bosonic Lagrangian and match it with the gauged $\mathcal{N}=4$ SUGRA coupled to SYM of [8], thereby identifying the correct mapping of field variables and parameters [1].

A major drawback of this simplistic model is of course that the $\mathcal{N}=4$ scenario forbids the appearance of chiral matter fields and is thus inappropriate for any phenomenological application. Still we believe the above program to be a useful check of the consistency of the approach in general, and hope it will carry over to $\mathcal{N}=1$ Calabi-Yau compactifications (see [1] for applications in this direction).

In 1] it was furthermore shown how the vacua of the effective action correspond to solutions of the ten-dimensional equations of motion. These solutions involve yet another background for a field strength whose potential is projected out of the type $\mathrm{I}^{\prime}$ spectrum, the purely internal and space-time filling components of the RR 5-form. It is related to the warp factor by the Einstein equations and is thus also neglected in the reduction. Clearly, it would be interesting to generalize the present method for constructing deformations of orientifold vacua to different T-dual versions of type I, as proposed in the SUGRA framework in 12 .

\section{The effective action with fluxes and open strings}

To cut short the long and technical story of deriving the effective action, we shall be slightly sketchy and jump over certain details in the following; the full computation can be found in [1]. Our starting point is the ten-dimensional type I Lagrangian

$$
\left(2 \kappa_{10}^{2}\right) \mathcal{L}_{\mathrm{I}}=e^{-2 \Phi}\left(R+4 \partial_{\mu} \Phi \partial^{\mu} \Phi\right)-\frac{1}{2}\left|\tilde{F}_{3}\right|^{2}-\tilde{\gamma} e^{-\Phi} \operatorname{tr}|\mathcal{F}|^{2},
$$

with $\tilde{\gamma}=\kappa_{10}^{2} / g_{10}^{2}$, and otherwise standard definitions for the fields. Most important for our purposes is the YM CS correction $\omega_{3}^{\mathrm{YM}}$ in the 3 -form field strength, $\tilde{F}_{3}=d C_{2}-$

$\tilde{\gamma} \omega_{3}^{\mathrm{YM}}$. After T-duality, this is to be compared to the truncation of the type IIB (pseudo-) Lagrangian

$$
\begin{aligned}
\left(2 \kappa_{10}^{2}\right) & \mathcal{L}_{\mathrm{IIB}}=e^{-2 \Phi}\left(R+4 \partial_{\mu} \Phi \partial^{\mu} \Phi-\frac{1}{2}\left|H_{3}\right|^{2}\right) \\
& -\frac{1}{2}\left(\left|F_{1}\right|^{2}+\left|F_{3}\right|^{2}+\frac{1}{2}\left|F_{5}\right|^{2}\right)-\frac{1}{2 \cdot 4 ! \cdot 3 ! \cdot 3 !} \epsilon^{i_{0} \cdots i_{9}}\left(C_{4}\right)_{i_{0} \cdots i_{3}}\left(d B_{2}\right)_{i_{4} i_{5} i_{6}}\left(d C_{2}\right)_{i_{7} i_{8} i_{9}}
\end{aligned}
$$

with $F_{3}=d C_{2}+C_{0} H_{3}, H_{3}=d B_{2}, F_{1}=d C_{0}$ and

$$
F_{5}=d C_{4}+\frac{1}{2} C_{2} \wedge d B_{2}-\frac{1}{2} B_{2} \wedge d C_{2} .
$$


The T-duality of the NSNS part of (3) is completely standard,

$$
G_{i j} \mapsto G^{i j}, \quad A_{\mu}^{i} \mapsto B_{\mu i}, \quad e^{2 \Phi} \mapsto G^{-1} e^{2 \Phi},
$$

$A_{\mu}^{i}$ being the $\mathrm{KK}$ vectors from the metric.

\section{$2.1 \quad$ T-duality of RR forms with open strings}

To perform the T-duality of the RR sector [13, i.e. of the kinetic term of $\tilde{F}_{3}$, it is very helpful to notice that the RR kinetic terms of type IIB can be put into a manifestly T-duality invariant form. To do so, replace the IIB RR and CS Lagrangian, the second line of (4), by the redundant form $\frac{1}{4}\left(\left|F_{1}\right|^{2}+\left|F_{3}\right|^{2}+\left|F_{5}\right|^{2}+\left|F_{7}\right|^{2}+\left|F_{9}\right|^{2}\right)$, plus impose $* F_{1}=F_{9}, * F_{3}=-F_{7}, * F_{5}=F_{5}$ after deriving the equations of motion. The new field strengths

$$
\sum_{p=0}^{4} F_{2 p+1}=e^{-B_{2}} \wedge \sum_{q=0}^{4} d D_{2 q}
$$

are defined through new RR potentials $D_{p}\left(C_{q}, B_{2}\right)$ which can be given explicitly. The $F_{p}$ and $D_{p}$ transform under the T-duality group $O(6,6, \mathbb{R})$ as spinors. Now one can apply the element of $O(6,6, \mathbb{R})$ that reflects all six circles (in some given order) to $F_{3}=d C_{2}$ and finds expressions like

$$
\left(d C_{2}\right)_{\mu i j}^{\{1,2\}} \mapsto-\frac{\sqrt{G}}{4 !} \epsilon^{i j k l m n}\left(d D_{4}\right)_{\mu k l m n}^{\{1,4\}},
$$

where the upper indices $\{p, q\}$ stand for the form-degree on $\mathbb{R}^{4} \times \mathbb{T}^{6}$, the numbers of internal and external indices. This looks qualitatively very much as expected, but the appearance of $D_{4}$ as opposed to $C_{4}$ is of course crucial here. Keeping track of the KK vectors that appear in the contractions in the kinetic terms, one finds that they reproduce the proper terms involving $B_{\mu i}$ in (7), and we obtain

$$
F_{3} \wedge * F_{3} \mapsto F_{9}^{\{3,6\}} \wedge * F_{9}^{\{3,6\}}+F_{7}^{\{2,5\}} \wedge * F_{7}^{\{2,5\}}+F_{5}^{\{1,4\}} \wedge * F_{5}^{\{1,4\}}+F_{3}^{\{0,3\}} \wedge * F_{3}^{\{0,3\}} .
$$

Note that the last term already corresponds to a RR 3-form flux, although $D_{2}^{\{0,2\}}$ is not in the spectrum. Adding the CS correction $\omega_{3}^{\mathrm{YM}}$ inside $\tilde{F}_{3}$ leads to

$$
\tilde{F}_{3} \wedge * \tilde{F}_{3} \mapsto \hat{F}_{9}^{\{3,6\}} \wedge * \hat{F}_{9}^{\{3,6\}}+\hat{F}_{7}^{\{2,5\}} \wedge * \hat{F}_{7}^{\{2,5\}}+\hat{F}_{5}^{\{1,4\}} \wedge * \hat{F}_{5}^{\{1,4\}}+\hat{F}_{3}^{\{0,3\}} \wedge * \hat{F}_{3}^{\{0,3\}}
$$

where the new CS corrected forms are

$$
\hat{F}_{3+2 p}^{\{p, p+3\}}=\left[e^{-B_{2}} \wedge \sum_{q=0}^{p}\left(d D_{2+2 q}+(-1)^{q(q-1) / 2} \gamma \star \omega_{3}\right)^{\{q, q+3\}}\right]^{\{p, p+3\}},
$$

with $\gamma=\sqrt{G}^{-1} \tilde{\gamma}$, and $\star$ denoting six-dimensional internal Hodge-duality. Dualizing the kinetic YM term by $A_{i}^{a} \mapsto A^{a i}$ and splitting ten-dimensional vectors into four-dimensional vectors and scalars produces kinetic terms for these, plus

$$
\sqrt{-g} \operatorname{tr}|\mathcal{F}|^{2} \mapsto \frac{1}{2} \sqrt{-g_{4}} G_{i j} G_{k l} f^{a b c} f^{a d e} A^{b i} A^{c k} A^{d j} A^{e l}+\cdots .
$$

This is the well known YM contribution to the scalar potential [14. 


\subsection{Modification due to 3-form fluxes}

So far we have produced an action that contains RR forms of all degrees. To compare it to the truncated type IIB Lagrangian we have to replace some of the forms of unconventional high degree by their Hodge duals. This can be done by a Lagrange multiplier procedure replacing $\hat{F}_{p}^{\{q, p-q\}}$ by $\hat{F}_{10-p}^{\{4-q, 6+q-p\}}$, which is standard to do without background fluxes. Before doing this, let us note that the formulation in which the BI action for the open string fields is usually given in type IIB also contains RR forms of all degrees, i.e. it provides a redundant version of the action, where duality relations must be imposed. Thus the redundant form is the right framework to investigate any extra modification coming from the BI action. Using the notation for the non-abelian BI action of [5], two types of terms are important for our purposes. The RR forms participate in additional Wess-Zumino interactions of the schematic form

$$
\begin{aligned}
\int \operatorname{tr}\left(C_{4}^{\{4,0\}} \mathrm{i}_{A} \mathrm{i}_{A} B_{2}\right) & \sim \int d^{4} x \sqrt{-g_{4}} \epsilon^{\mu \nu \rho \sigma}\left(C_{4}\right)_{\mu \nu \rho \sigma} H_{i j k} \operatorname{tr}\left(A^{i} A^{j} A^{k}\right), \\
\int \operatorname{tr}\left(\mathrm{P}\left[\mathrm{i}_{A} \mathrm{i}_{A} C_{6}\right]\right) & \sim \int d^{4} x \sqrt{-g_{4}} \epsilon^{\mu \nu \rho \sigma}\left(d C_{6}\right)_{\mu \nu \rho \sigma i j k} \operatorname{tr}\left(A^{i} A^{j} A^{k}\right),
\end{aligned}
$$

where $\mathrm{i}_{A} \mathrm{i}_{A} B_{2}=A^{j} A^{i} B_{i j}$, etc. The CS interactions of the component $C_{4}^{\{4,0\}}$, nondynamical in four dimensions, are of central importance since its ten-dimensional equation of motion determines the tadpole cancellation or RR charge conservation constraint. It is then crucial that the two terms combine into an interaction term of the form $\left(F_{7}\right)_{\mu \nu \rho \sigma i j k}^{\{4,3\}} \operatorname{tr}\left(A^{i} A^{j} A^{k}\right)+\cdots$, and therefore there is no additional contribution to the tadpole condition after dualizing $F_{7}^{\{4,3\}}$ to $F_{3}^{\{0,3\}}$. It then follows that the RR charge effectively carried by the background flux remains unmodified compared to the case of trivial open string fields [9],

$$
N_{\text {flux }}=\frac{1}{2 \kappa_{10}^{2} \mu_{3}} \int F_{3}^{\{0,3\}} \wedge H_{3}^{\{0,3\}} .
$$

I.e. in contrast to (9), there is no hat on $F_{3}$. We come back to the physical effects of this at the end of the section. The second term we need comes from the BI action itself and involves the NSNS 3-form flux,

$$
\frac{i}{3 g_{10}^{2} \sqrt{G}} e^{-\Phi} \operatorname{tr}\left(A^{i} A^{j} A^{k}\right)\left(H_{3}\right)_{i j k}=-\frac{1}{2 \kappa_{10}^{2}} \frac{1}{3 !} \gamma e^{-\Phi} \omega_{3}^{i j k}\left(H_{3}\right)_{i j k} .
$$

When we add the brane tension to the effective potential, this term has to be added as well. Together with (13), it ensures the positivity of the potential.

We can now go ahead with the procedure to replace the RR forms of higher degree. For the details we again refer to [1]. One imposes the Bianchi-identites of the various field strengths through Lagrange multipliers, and then integrates out the original forms through their equations of motion, leaving the Lagrange multipliers, which roughly are the Hodge-duals of the original fields. This allows us to put the action into standard form

$$
\hat{F}_{9}^{\{3,6\}} \wedge * \hat{F}_{9}^{\{3,6\}}+\hat{F}_{7}^{\{2,5\}} \wedge * \hat{F}_{7}^{\{2,5\}} \rightarrow F_{1}^{\{1,0\}} \wedge * F_{1}^{\{1,0\}}+F_{3}^{\{2,1\}} \wedge * F_{3}^{\{2,1\}}+2\left(2 \kappa_{10}^{2}\right) \mathcal{L}_{\mathrm{CS}}
$$

with $F_{1}, F_{3}$ as in type IIB. The extra piece in the Lagrangian that is generated along the way consists of several CS terms and reads, after reducing to four dimensions,

$$
\left(2 \kappa_{4}^{2}\right) \mathcal{L}_{\mathrm{CS}}=-\frac{1}{4} \tilde{\gamma} \epsilon^{\mu \nu \rho \sigma}\left(C_{0} \tilde{\mathcal{F}}_{\mu \nu}^{a} \tilde{\mathcal{F}}_{\rho \sigma}^{a}-2\left(F_{j \mu \nu}-C_{0} H_{j \mu \nu}\right)\left(A^{a j} \tilde{\mathcal{F}}_{\rho \sigma}^{a}+\frac{1}{2} A^{a j} A^{a i} H_{i \rho \sigma}\right)\right)
$$




$$
+\frac{1}{4 \cdot 4 !} \epsilon^{\mu \nu \rho \sigma} \epsilon^{i j k l m n} C_{i j k l} F_{m \mu \nu} H_{n \rho \sigma} .
$$

The first line is the new CS action that couples open string and KK vector fields and scalars, while the second line is the type IIB bulk CS term subject to projection with $\Omega^{\prime}$. In the presence of fluxes the bulk CS term gets additional contributions, not displayed in (15), which can be obtained by demanding invariance under gauge-symmetry, see [1. Note that $\tilde{\mathcal{F}}_{\mu \nu}$ refers to redefined gauge fields $\tilde{A}_{\mu}^{a}=A_{\mu}^{a}-A^{a i} B_{\mu i}$. We find it a very satisfactory check of the methods used that (15) matches the expressions given in [8] for the coupled $\mathcal{N}=4$ SUGRA-SYM Lagrangian.

Finally, we can deduce the additional terms that arise when projecting the type IIB Lagrangian (44) in the simultaneous presence of NSNS and RR 3-form fluxes with the modified world sheet parity $\Omega^{\prime}$. We just note two significant examples. The kinetic terms for the axionic scalars that descend from the internal RR 4-form $C_{4}$ in (5) are modified by

$$
\begin{aligned}
& \partial_{\mu}\left(C_{4}\right)_{i j k l}-2\left(B_{2}\right)_{\mu[i}\left(d C_{2}+2 \gamma \star \omega_{3}\right)_{j k l]}+\gamma\left(\star \omega_{3}\right)_{\mu i j k l} \rightarrow \\
& \partial_{\mu}\left(C_{4}\right)_{i j k l}-2\left(B_{2}\right)_{\mu[i}\left(d C_{2}+2 \gamma \star \omega_{3}\right)_{j k l]}+\gamma\left(\star \omega_{3}\right)_{\mu i j k l}+2\left(C_{2}\right)_{\mu[i}\left(d B_{2}\right)_{j k l]},
\end{aligned}
$$

i.e. extra Stückelberg mass terms appear in $\left|\hat{F}_{5}^{\{1,4\}}\right|^{2}$. The scalar potential, which was already present through the "kinetic term" of $\hat{F}_{3}^{\{0,3\}}$, is extended to

$$
\left|\left(d C_{2}+\gamma \star \omega_{3}\right)^{\{0,3\}}\right|^{2} \rightarrow\left|\left(d D_{2}+\gamma \star \omega_{3}\right)^{\{0,3\}}\right|^{2}+e^{-2 \Phi}\left|H_{3}^{\{0,3\}}\right|^{2}=\left|\hat{G}_{3}^{\{0,3\}}\right|^{2},
$$

i.e. the (CS corrected) complex IIB 3-form flux $\hat{G}_{3}^{\{0,3\}}$ is completed. As already mentioned, one also has to add the tension of the localized sources (D3-branes and O3-planes), including the extra non-abelian term (14), to the flux energy

$$
\left|\hat{G}_{3}^{\{0,3\}}\right|^{2}-\frac{1}{18} e^{-\Phi} \epsilon^{i j k l m n}\left(F_{3}\right)_{i j k}\left(H_{3}\right)_{l m n}-\frac{4 i}{3} \gamma e^{-\Phi} \operatorname{tr}\left(A^{i} A^{j} A^{k}\right)\left(H_{3}\right)_{i j k}=2\left|\hat{G}_{3}^{\mathrm{ISD}}\right|^{2},
$$

ISD indicating the imaginary-self-dual part of $\hat{G}_{3}^{\{0,3\}}$ under internal Hodge duality [9. The second term is the contribution of the tension and it is crucial therefore that (13) did not contain $\hat{F}_{3}^{\{0,3\}}$. Otherwise (18) would not have come out positive definite. This would then have also implied that even if $\hat{G}_{3}^{\text {ISD }}=0$, the typical coupling that drives the dielectric effect would not have vanished, contrary to expectation.

\subsection{Some consequences}

To compare with the results of gauged supergravity, we transform to the four-dimensional Einstein-frame, yielding the full effective potential in agreement with [8],

$$
\left(2 \kappa_{4}^{2}\right) \mathcal{V}_{\mathrm{eff}}=\frac{e^{\Phi}}{\sqrt{G}}\left|\left(G_{3}+\gamma \star \omega_{3}\right)^{\mathrm{ISD}}\right|^{2}+\frac{\tilde{\gamma} e^{\Phi}}{2 G} G_{i j} G_{k l} f^{a b c} f^{a d e} A^{b i} A^{c k} A^{d j} A^{e l} .
$$

It is positive definite and of the no-scale type. It has been stressed in [15] that this is consistent with the absence of solutions to the ten-dimensional equations of motion with a positive four-dimensional cosmological constant, due to the fact that the volume modulus does not have a stable minimum. Indeed the global minima of the potential, its Minkowski vacua, are characterized by $\left(G_{3}+\gamma \star \omega_{3}\right)^{\mathrm{ISD}}=f^{a b c} A^{i b} A^{j c}=0$. Since these conditions scale 
trivially under rescaling of the total volume $R^{6}, R$ is a free modulus. This also provides us with the opportunity to meet the self-consistency requirement (2) by simply choosing

the free parameter $R$ large in terms of $\sqrt{\alpha^{\prime}}$. On the other hand, one eventually has to break up the no-scale structure to stabilize the volume, possibly by higher derivative [16] or non-perturbative [10] corrections to the effective action. Then (2) has to be met as a restriction on the vacuum, otherwise the scalar potential (19) is not reliable.

\section{Acknowledgements}

B.K. is grateful to the organizers of the 36th International Symposium Ahrenshoop on the Theory of Elementary Particles, Recent Developments in String/M-Theory and Field Theory, who created an inspiring atmosphere for a great workshop. The work of B. K. was supported by the German Science Foundation (DFG) and in part by funds provided by the U.S. Department of Energy (D.O.E.) under cooperative research agreement \#DFFC02-94ER40818. The work of M. B. and M. H. was supported in part by I.N.F.N., by the E.C. RTN programs HPRN-CT-2000-00122 and HPRN-CT-2000-00148, by the INTAS contract 99-1-590, by the MURST-COFIN contract 2001-025492, by the NATO contract PST.CLG.978785 and by the National Science Foundation under Grant No. PHY99-07949. Moreover, M. B. was supported by a Marie Curie Fellowship, contract \# HPMF-CT-2001-01311 and by the Wenner-Gren Foundations, and M. H. by the German Science Foundation (DFG).

\section{References}

[1] M. Berg, M. Haack and B. Körs, Nucl. Phys. B 669 (2003) 3 hep-th/0305183.

[2] A. Strominger, Nucl. Phys. B 274 (1986) 253.

[3] B. de Wit, D. J. Smit and N. D. Hari Dass, Nucl. Phys. B 283 (1987) 165.

[4] J. Polchinski and A. Strominger, Phys. Lett. B 388 (1996) 736 hep-th/9510227.

[5] R. C. Myers, JHEP 9912 (1999) 022 hep-th/9910053.

[6] S. Kachru, M. B. Schulz and S. Trivedi, JHEP 0310 (2003) 007 hep-th/0201028.

[7] A. R. Frey and J. Polchinski, Phys. Rev. D 65 (2002) 126009 hep-th/0201029.

[8] R. D'Auria, S. Ferrara and S. Vaula, New J. Phys. 4 (2002) 71 hep-th/0206241; S. Ferrara and M. Porrati, Phys. Lett. B 545 (2002) 411, hep-th/0207135; R. D'Auria, S. Ferrara, M. A. Lledo and S. Vaula, Phys. Lett. B 557 (2003) 278 hep-th/0211027; R. D'Auria, S. Ferrara, F. Gargiulo, M. Trigiante and S. Vaula, JHEP 0306 (2003) 045 hep-th/0303049.

[9] S. B. Giddings, S. Kachru and J. Polchinski, Phys. Rev. D 66 (2002) 106006 hep-th/0105097.

[10] S. Kachru, R. Kallosh, A. Linde and S. P. Trivedi, Phys. Rev. D 68 (2003) 046005 hep-th/0301240. 
[11] R. Blumenhagen, D. Lüst and T. R. Taylor, Nucl. Phys. B 663 (2003) 319 hep-th/0303016; J. F. G. Cascales and A. M. Uranga, JHEP 0305 (2003) 011 hep-th/0303024; P. G. Camara, L. E. Ibanez and A. M. Uranga, hep-th/0311241; J. F. G. Cascales, M. P. G. del Moral, F. Quevedo and A. M. Uranga, hep-th/0312051.

[12] C. Angelantonj, S. Ferrara and M. Trigiante, JHEP 0310 (2003) 015 hep-th/0306185.

[13] M. Fukuma, T. Oota and H. Tanaka, Prog. Theor. Phys. 103 (2000) 425 hep-th/9907132; S. F. Hassan, Nucl. Phys. B 583 (2000) 431, hep-th/9912236; S. F. Hassan and R. Minasian, hep-th/0008149.

[14] E. Witten, Phys. Lett. B 155 (1985) 151.

[15] S. P. de Alwis, hep-th/0307084.

[16] K. Becker, M. Becker, M. Haack and J. Louis, JHEP 0206 (2002) 060 hep-th/0204254. 